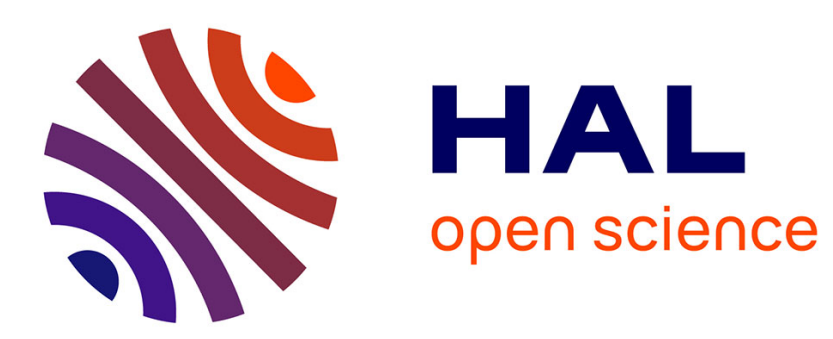

\title{
Wigner Quantization of Hamilton-Dirac Systems
}

T. S. Ratiu, O. G. Smolyanov

\section{- To cite this version:}

T. S. Ratiu, O. G. Smolyanov. Wigner Quantization of Hamilton-Dirac Systems.

Doklady Mathematics, 2015, 91 (1), pp.114-116. 10.1134/S1064562415010287 . hal-01398473

\section{HAL Id: hal-01398473 \\ https://hal.science/hal-01398473}

Submitted on 17 Nov 2016

HAL is a multi-disciplinary open access archive for the deposit and dissemination of scientific research documents, whether they are published or not. The documents may come from teaching and research institutions in France or abroad, or from public or private research centers.
L'archive ouverte pluridisciplinaire HAL, est destinée au dépôt et à la diffusion de documents scientifiques de niveau recherche, publiés ou non, émanant des établissements d'enseignement et de recherche français ou étrangers, des laboratoires publics ou privés. 


\title{
Wigner Quantization of Hamilton-Dirac Systems
}

\author{
T. S. Ratiu ${ }^{a}$ and O. G. Smolyanov ${ }^{b}$ \\ Presented by Academician V.V. Kozlov February 4, 2014
}

This paper describes the states of quantum versions of finite-dimensional Hamilton-Dirac systems in terms of (analogues of) the Wigner function (various definitions of Wigner functions for classical Hamiltonian systems can be found in [8]). We give a system of equations, which we call the Moyal-Dirac system, describing the evolution of this Wigner function. The passage from the Moyal equation describing the evolution of the quantum version of a classical Hamiltonian system to the Moyal-Dirac system describing the evolution of the quantum version of the corresponding Hamilton-Dirac system is similar to the passage from the Liouville equation describing the evolution of a classical Hamiltonian system to the Liouville-Dirac system of equations describing the evolution of the corresponding Hamilton-Dirac system. Thus, our Moyal-Dirac system is a generalization of both the Liouville-Dirac system and the Moyal equation.

If a Hamilton-Dirac system is infinite-dimensional, then, instead of the Wigner function, the socalled Wigner measure [7] should be used, because, by virtue of Weil's well-known theorem, on an infinitedimensional (topological vector) space, there exists no analogue of the standard Lebesgue measure, i.e., no nonzero $\sigma$-finite locally finite countably additive translation-invariant Borel measure. In the finitedimensional case, the density of the Wigner measure with respect to the Lebesgue measure is the Wigner function.

Hamilton-Dirac systems were introduced by Dirac (see [4] and the references therein), who called them generalized Hamiltonian systems. They are also known as constrained Hamiltonian systems; such systems can be regarded as objects dual to Lagrangian systems with nonholonomic constraints (Lagrangian

\footnotetext{
${ }^{a}$ Mathematics Section and Bernoulli Center, École Polytechnique Féedérale de Lausanne, Station 8, Lausanne, $\mathrm{CH}-1015$ Switzerland e-mail: tudor.ratiu@epfl.ch

${ }^{b}$ Mechanics and Mathematics Faculty, Moscow State University, Moscow, 119991 Russia e-mail:smolyanov@yandex.ru
}

system with holonomic constraints bear no relation to constrained Hamiltonian systems).

Hamilton-Dirac systems arise under the application of the Legendre transform to an unconstrained Lagrangian system with degenerate Lagrange function (for such Lagrange functions, which are also called singular, the Legendre transform is noninvertible). It is worth mentioning that to Lagrangian systems with nondegenerate Lagrange function and nonholonomic constraints the Legendre transform assigns unconstrained Hamiltonian systems with degenerate Hamilton function (for which the Legendre transform is again noninvertible).

In what follows, we emphasize the algebraic aspects of the theory and omit analytical details.

\section{NOTATION AND TERMINOLOGY}

The definitions and notation used in this paper can largely be found in our paper [12]. A Hamilton-Dirac system is a quadruple $(E, \mathbb{J}, \mathcal{H}, \psi)$, where $E$ is a real Hausdorff locally convex space (LCS), $\mathbb{J} \in \mathscr{L}\left(E^{\prime}, E\right)$ is the vector space of all continuous linear mappings of the topological dual $E^{\prime}$ of $E^{\prime}$ to the space $E, \mathbb{J} *=-\mathbb{J}, \mathscr{H}^{*}$ is a real- or complex-valued function on $E$, called the Hamilton function (of the Hamilton-Dirac system), and $\psi$ is a smooth mapping of the LCS $E$ to an auxiliary LCS $Z_{\psi}$. The pair $(E, \mathbb{J})$ is a symplectic LCS, called the phase space of the Hamilton-Dirac system.

By $\{\cdot, \cdot\}$ we denote the Poisson bracket; we define it not only for scalar functions but also for functions taking values in an LCS (see [12]).

Given smooth numerical functions $f$ and $g$ on $E$, their Poisson bracket is defined by $\{f, g\}:=$ $f^{\prime}(x)\left(\sqrt{ } g^{\prime}(x)\right)$. If $G_{1}$ and $G_{2}$ are LCSs, $f, g \in C^{\infty}(E, \mathbb{C})$, $a \in G_{1}$, and $b \in G_{2}$, then the functions $a \otimes f \in C^{\infty}(E$, $\left.G_{1}\right)$ and $b \otimes g \in C^{\infty}\left(E, G_{2}\right)$ are defined by $(a \otimes f)(x):=$ $f(x) a G_{1}$ and $(b \otimes g)(x):=g(x) b \in G_{2}$ for $x \in E$. The Poisson bracket $\{a \otimes f, b \otimes g\} \in C^{\infty}\left(E, G_{1} \otimes G_{2}\right)$ is defined by $\{a \otimes f, b \otimes g\}(x):=\{f, g\}(a \otimes b)$, where $\{f, g\}$ is the Poisson bracket of the scalar functions. The mapping $(a \otimes f, b \otimes g) \mapsto\{a \otimes f, b \otimes g\}$ can be extended to a bilinear mapping $(\varphi, \psi) \mapsto\{\varphi, \psi\}$ of the product of the space $F_{1}:=G_{1} \otimes C^{\infty}(E, \mathbb{C}) \subset C^{\infty}\left(E, G_{1}\right)$ and the 
space $F_{2}:=G_{2} \otimes C^{\infty}(E, \mathbb{C}) \subset C^{\infty}\left(E, G_{2}\right)$ to the space $C^{\infty}\left(E, G_{1} \otimes G_{2}\right)$. Clearly, the Poisson bracket $\{\cdot, \cdot\}$ thus defined is skew-symmetric. If $G_{3}$ is yet another LCS, $F_{3}:=G_{3} \otimes \otimes C^{\infty}(E, \mathbb{C}) \subset C^{\infty}\left(E, G_{3}\right), f \in F_{1}, g \in F_{2}$, and $h \in F_{3}$, then $\{f,\{g, h\}\}(x)+\{g,\{h, f\}\}(x)+\{h,\{f, g\}\}(x)=0$ in $G_{1} \otimes \otimes G_{2} \otimes G_{3}$ for any $x \in E$ (by virtue of the associativity of algebraic tensor product). For the product $f \cdot g$ defined by $(f \cdot g)(x):=f(x) \otimes g(x)$, the Leibniz rule $\{f, g \cdot h\}=\{f, g\} \cdot h+g \cdot\{f, h\} \in C^{\infty}\left(E, G_{1} \otimes G_{2} \otimes G_{3}\right)$ also holds. Thus, the Poisson bracket of functions taking values in an LCS has the same properties as the Poisson bracket of scalar functions.

We set $\mathfrak{m}_{\psi}:=\{x \in E \mid \psi(x)=0\}$; it is assumed in what follows that 0 is a regular value of $\psi$ and $x \in \mathfrak{m}_{\psi}$ implies $\{\psi, \psi\}(x)=0$ and $\{\mathscr{H}, \psi\}(x)=0$.

The Liouville-Dirac system of equations for a Hamilton-Dirac system $(E, \mathbb{J}, \mathcal{H}, \psi)$ is the system

$$
\begin{gathered}
\dot{F}(t)=\left\{\mathscr{H}_{\lambda}, F(t)\right\}, \\
\{\psi, F(t)\}=0
\end{gathered}
$$

with respect to an unknown function $F(\cdot)$ of a real argument $t$ taking values in the set of smooth functions on $E$; here, $\mathscr{H}_{\lambda}(t):=\mathscr{H}+\lambda(t) \psi$ and $\lambda$ is an arbitrary function of the same argument taking values in $Z_{\psi}^{\prime}$ (in Dirac's book [4], the function $\mathcal{H}_{\lambda}$ is called a generalized Hamiltonian).

By a solution of the Liouville-Dirac system we mean a function $F(\cdot)$ satisfying system (1) on the set $\mathfrak{m}_{\psi}$.

\section{WIGNER FUNCTIONS AND MEASURES}

In what follows, we assume that $Q$ and $P$ are copies of a real Hilbert space $H$, and $Q^{\prime}=P, P^{\prime}=Q$, and $E$ is identified with the Hilbert sum of the Hilbert spaces $Q$ and $P$. The elements $h \in E$ are identified with linear continuous functionals $E \ni x \mapsto(x, h)$ on $E$; by $\hat{h}$ we denote the pseudodifferential operator with symbol $h$ defined on an appropriate space of functions or measures on $Q$.

Definition 1. If $h=q^{h}+p^{h}$, where $q^{h} \in Q, p^{h} \in P$, $\psi$ is a suitable function on $Q$, and $\operatorname{dim} Q=\operatorname{dim} P=n$, then $\hat{h} \psi$ is the function on $Q$ defined by

$$
=\frac{1}{(2 \pi)^{n}} \int_{P Q} \int_{Q}^{i p\left(q_{1}-q\right)}\left(\left(q^{h}, p\right)+\left(p^{h}, q_{1}\right)\right) \psi\left(q_{1}\right) d q_{1} d p .
$$

Definition 2. The Wigner measure generated by a density operator $T$ is the (generalized) measure $W^{T}$ on the phase space $E$ defined by

$$
\begin{gathered}
\int_{Q \times P} \exp \left[i\left(\left(p_{1}, q_{2}\right)+\left(q_{1}, p_{2}\right)\right)\right] W^{T}\left(d q_{1}, d p_{1}\right) \\
=\operatorname{tr} T e^{-i\left(\hat{q}_{2}+\hat{p}_{2}\right)} .
\end{gathered}
$$

Remark 1. The function $(q, p) \mapsto \operatorname{tr} T e^{i(\hat{q}+\hat{p})}$ is called the Weyl function; thus, the Wigner measure is the (inverse) Fourier transform of the Weyl function.

If $\mu$ is a nonnegative Borel measure on $E$, then the density of the Wigner measure $W^{T}$ with respect to $\mu$ is called the Wigner $\mu$-function and denoted by $W_{\mu}^{T}$; if $\operatorname{dim} E<\infty$ and $\mu$ is the standard Lebesgue measure, then the Wigner $\mu$-function is called the Wigner function.

Remark 2. We have $e^{\alpha(\hat{q}+\hat{p})}=\left(\widehat{e^{\alpha(q+p)}}\right)$; thus, in this case, the exponential of the pseudodifferential operator with symbol $q+p$ coincides with the pseudodifferential operator whose symbol is the exponential of the former symbol (i.e., of the function $q+p$ ); we emphasize that a similar assertion for symbols not being linear functions is false.

\section{THE MOYAL-DIRAC EQUATIONS: THE FINITE-DIMENSIONAL CASE}

In this section, we assume that $\operatorname{dim} H<\infty$. For each $t \in[0, \infty)$, let $T(t)$ be the density operator describing the state at the moment of time $t$ of the quantum system obtained by quantizing the Hamilton-Dirac system $(E, \mathbb{J}, \mathcal{H}, \psi)$. Let $W_{T}(t)(\cdot, \cdot):=W_{T(t)}(\cdot, \cdot)$ be the Wigner function corresponding to the density operator $T(t)$, and let $\mathscr{L}_{\mathscr{H}_{\lambda}}(x):=\left\{f, \mathscr{H}_{\lambda}\right\}(x)$ for $f \in C^{\infty}(E, \mathbb{C})$ ( $\mathscr{L}_{\mathscr{H}_{\lambda}}$ is called the Liouville operator).

Theorem 1. There exists a function $\lambda:[0, \infty) \rightarrow Z_{\psi}^{\prime}$ for which $W_{T}(\cdot)$ satisfies the Moyal-Dirac system of equations

$$
\begin{gathered}
\dot{W}_{T}(t)(q, p)=2 \sin \frac{1}{2} \mathscr{L}_{\mathscr{H}_{\lambda}}\left(W_{T}(t)\right)(q, p), \\
\left\{\psi, W_{T}(t)\right\}(q, p)=0
\end{gathered}
$$

on the set of those $(q, p) \in$ Efor which $\psi(q, p)=0$. Here,

$$
\sin a \mathscr{L}_{G}:=\sum_{n=1}^{\infty} \frac{a}{(2 n-1) !} \mathscr{L}_{G}^{(n-1)}
$$

and $\left(\mathscr{L}_{G}^{(n)} F\right)(x):=F^{(n)} \rrbracket^{\otimes n} G^{(n)}(x)$.

\section{INFINITE-DIMENSIONAL MOYAL-DIRAC EQUATIONS}

As mentioned above, in the case where $\operatorname{dim} H=\infty$, instead of Wigner functions, Wigner measures, or Wigner $\mu$-functions, should be used. In the case of unconstrained infinite-dimensional Hamiltonian systems, the passage from equations for functions to equations for measures is straightforward, because the Liouville operator can be naturally defined on measure spaces; this fact can be regarded as an infinitedimensional version of Liouville's theorem on the 
preservation of phase volume (which does not exist in infinite-dimensional spaces) under the canonical transformations.

In the case of infinite-dimensional HamiltonDirac systems, the passage to equations with respect to measures requires attaching meaning to the assertion that the Moyal-Dirac system (2) is satisfied on the set $\{(q, p) \mid \psi(q, p)=0\}$. This can be done by replacing the passage from a Wigner function defined on the entire phase space to its restriction to the submanifold $\{(q, p) \mid \psi(q, p)=0\}$ by the passage from a Wigner measure defined on $E$ to the corresponding surface measure.

\section{FEYNMAN FORMULAS}

A Feynman formula is a representation of a solution of an evolution differential equation or some object related to this equation (e.g., the trace of the operator on the right-hand side of the equation) in terms of the limit of integrals over Cartesian powers of some space, e.g., the configuration or phase space of the corresponding Hamiltonian system, as the exponent tends to infinity.

To obtain a Feynman formula for a solution of the Moyal-Dirac system (2) describing the quantum system corresponding to a (classical) Hamilton-Dirac system, we note that the Moyal-Dirac system coincides with a system of Schrödinger-type equations for the extended Hamilton-Dirac system $\left(E_{\text {ext }}, \mathbb{J}_{\text {ext }}, \mathscr{H}_{\text {ext }}\right.$, $\left.\psi_{\text {ext }}\right)$, where $E^{\text {ext }}:=E_{Q} \times E_{P}, E_{Q}$ and $E_{P}$ are two copies of the space $E, \mathbb{J}_{\text {ext }}(h, k):=(k,-h)$ for $(h, k) \in E_{Q} \times E_{P}$, $\psi_{\mathrm{ext}}(k):=\psi^{\prime}(k) \rrbracket k$, and

$$
\mathcal{H}^{\text {ext }}(h, k):=2 \sum_{n=1}^{\infty} \frac{1}{2^{2 n-1}(2 n-1) !} \mathcal{H}_{\lambda}^{(n)}(h) \rrbracket^{\otimes n}(k)^{\otimes n} .
$$

Theorem 2. If $W_{T}(\cdot)$ is a solution of the Moyal-Dirac system (2) and $\psi(q, p)=0$, then the following Feynman formula is valid:

$$
V_{T}(t)(q, p)=\left(\lim _{n \rightarrow \infty}\left(\widehat{\exp \left(i \frac{t}{n} \mathcal{H}^{\text {ext }}\right)}\right)^{n} W_{T}(0)\right)(q, p)
$$

Here, $\widehat{\exp \left(\frac{i-t}{n} \mathscr{H}^{\text {ext }}\right)}$ denotes the pseudodifferential operator with symbol $\overline{\exp \left(i \frac{t}{n} \mathscr{H}^{\text {ext }}(\cdot)\right)}$; its definition, which is similar to Definition 1 given above, can be found in [12].

\section{ACKNOWLEDGMENTS}

This work was supported by the Government of Russian Federation, decree no. 220 (contract no. 11.G34.31.0054 between Ministry of Education and Science of Russian Federation and Lomonosov Moscow State University). T.S. Ratiu acknowledges the support of Swiss National Science Foundation, grant no. 200021-140238. Smolyanov acknowledges the support of the Bernoulli Center, Federal Polytechnic School of Lausanne, where a major part of the work was completed, and of the Russian Foundation for Basic Research (project no. 14-01-00516).

\section{REFERENCES}

1. R. Abraham and J. E. Marsden, Foundations of Mechanics, 2nd ed. (Addison-Wesley, Reading, Mass., 1978).

2. S. Albeverio, O. G. Smolyanov, and A. Yu. Khrennikov, Dokl. Math. 64, 321-325 (2001).

3. P. R. Chernoff and J. E. Marsden, Some Basic Properties of Infinite Dimensional Hamiltonian Systems (SpringerVerlag, Berlin, 1974).

4. P. A. M. Dirac, Lectures on Quantum Mechanics (Yeshiva Univ., New York, 1964; Mir, Moscow, 1968).

5. K.-J. Engel and R. Nagel, One-Parameter Semigroups for Linear Evolution Equations (Springer, New York, 2000).

6. J. Gough, T. S. Ratiu, and O. G. Smolyanov, Dokl. Math. 89, 68-71 (2014).

7. V. V. Kozlov and O. G. Smolyanov, Dokl. Math. 84, 571-575 (2011).

8. V. V. Kozlov and O. G. Smolyanov, Theory Prob. Its Appl. 51 (1), 168-181 (2007).

9. J. E. Marsden and T. S. Ratiu, Introduction to Mechanics and Symmetry (Springer-Verlag, Berlin, 1994).

10. J.-P. Ortega and T. S. Ratiu, Momentum Maps and Hamiltonian Reduction (Birkhäuser, Boston, Mass., 2004).

11. T. S. Ratiu and O. G. Smolyanov, Dokl. Math. 87, 289292 (2013).

12. T. S. Ratiu and O. G. Smolyanov, Dokl. Math. 91, (2015).

Translated by O. Sipacheva 